\title{
Un nouveau regard sur l'affichage urbain : le passage Saint Rémy à Bordeaux
}

Mahdi Amri, Anne-Laure Cano, Marie-Julie Catoir, Emilie Lechenaut et Martine Versel

\section{CpenEdition}

12 Journals

Édition électronique

URL : http://journals.openedition.org/communicationorganisation/367

DOI : 10.4000/communicationorganisation.367

ISSN : $1775-3546$

Éditeur

Presses universitaires de Bordeaux

Édition imprimée

Date de publication : 1 décembre 2007

Pagination : 124-148

ISSN : 1168-5549

\section{Référence électronique}

Mahdi Amri, Anne-Laure Cano, Marie-Julie Catoir, Emilie Lechenaut et Martine Versel, « Un nouveau

regard sur l'affichage urbain : le passage Saint Rémy à Bordeaux », Communication et organisation [En ligne], 32 | 2007, mis en ligne le 01 décembre 2010, consulté le 30 avril 2019. URL : http://

journals.openedition.org/communicationorganisation/367; DOI : 10.4000/

communicationorganisation.367 
Analyses

\title{
Résumé
}

Cet article aborde l'affichage urbain à travers l'étude d'un Passage (le Passage St Rémi à Bordeaux) qui est caractérisé par un télescopage d'affiches, d'enseignes institutionnelles et d'affichages sauvages. C'est à travers la sémiotique de l'objet et son cadre théorique autour de la tensivité du lisible et du visible que nous avons opéré. Le point de départ de notre étude, qui favorise le corps phénoménologique et permet de saisir l'articulation d'une manipulation spécifique entre objets, nous engage de nous déplacer en doublant le postulat perceptif par celui de la pulsion (le pousse-à-voir psychanalytique). Ainsi l'affichage urbain serait une quête sans suspension possible, sans butée de l'au-delà du visible et entretiendrait à vide, un regard qui ne verrait plus grand-chose.

\section{Mots-clés}

Affichage urbain, sémiotique et épistémologie, psychanalyse.

\begin{abstract}
This article deals with urban billsticking through the study of a peculiar passage-way (the Passage St Remi in Bordeaux) which is characterized by an overlap of institutional signs and illegal billsticking. From a theoretical point of view, the approach is based on the semiotics of the object and more specifically on the tensivity between legible and visible. This starting point, which favourize the phenomenological body and helps us to understand the articulation of a specific manipulation between the objects, makes it possible to move and to double the perceptive postulate with the instinct one (the psychoanalytic "pousse-à-voir"). Thus, urban billsticking would be a quest with no suspension possible, no stop for what is beyond the visible, and would keep a glance that would not see anything going.
\end{abstract}

\section{Key-Words}

Urban publicity, semiotic, epistemology, psychanalysis.

Mahdi Amri, Anne-Laure Cano, Marie-Julie Catoir et Emilie Lechenaut sont doctorants en Sciences de l'Information et de la Communication à l'Université Michel de Montaigne - Bordeaux 3, au sein du laboratoire IMAGINES, groupe de recherche sur les images, histoires et sociétés, dans cette Université.

Martine Versel est Maître de conférences en Sciences de l'Information et de la Communication à l'Université Michel de Montaigne - Bordeaux 3. Elle est chercheur au sein du laboratoire IMAGINES, groupe de recherche sur les images, histoires et sociétés, dans cette Université. 


\section{Un nouveau regard sur l'affichage urbain : le passage Saint Rémy à Bordeaux Mahdi Amri, Anne-Laure Cano, Marie-Julie Catoir, Emilie Lechenaut, Martine Versel \\ amrimahdi@yahoo.fr annelaure.cano@gmail.com mariecatoir@voila.fr elechenaut@hotmail.com versel@u-bordeaux3.fr}

Nous proposons ici, un développement de toutes les étapes d'une recherche sur le thème de l'affichage urbain. Précisons d'emblée que dans cette recherche, nous avons toutefois eu un parti pris initial: celui d'une approche sémiotique ; non pas pour faire une stricte application des outils sémiotiques, mais au contraire, afin de les confronter à notre problématique. En effet, cette confrontation nous a permis d'ouvrir des hypothèses, mais aussi une réflexion sur les enjeux de lisibilité et de visibilité.

C'est notamment en étudiant ces enjeux et à partir d'un protocole que nous avons construit, que nous avons pu mettre en lumière un jeu de tension entre lisibilité et visibilité. Un déplacement vers l'approche psychanalytique nous a dès lors semblé nécessaire et pertinent. Ce déplacement méthodologique nous a en outre permis d'approfondir notre analyse sur les jeux de tensions entre les affiches, les supports et le public. Nous avions également la volonté, dès le départ, de nous confronter aux limites pratiques d'une analyse sémiotique.

Il convient enfin de noter, avant d'ouvrir notre propos, que l'urbanité et l'affichage urbain constituent un champ de recherche à part entière. Cependant, dans le corps de notre travail, nous utiliserons ces termes dans leur sens d'usage commun, comme catégories sémantiques, afin de réaliser notre analyse sémiotique.

\section{Quelques considérations sur l'affichage urbain}

Dans le décor urbain des villes contemporaines se trouve une multitude d'objets plantés, fixés, qui permettent de lire la ville dans sa propre matérialité. Ils en délimitent les contours, individualisent certains secteurs, servent de repère pour qualifier des lieux. Ils offrent notamment, par l'effet combiné de leur emplacement et de leur message, la possibilité d'identifier les principales lignes de force selon lesquelles s'organise l'espace social. Ces objets sont les affiches urbaines. 
Analyses

Elément majeur du décor urbain et du spectacle de la rue, l'affiche est à appréhender dans la fluidité spatiale de la ville ${ }^{1}$. Comme le souligne Alain Mons, elle fait partie de la mobilité apparente de la ville tout en constituant une pièce maitresse de cette architecture sociale, mouvante et quotidienne. Il semblerait d'ailleurs qu'elle soit perçue comme un arrêt possible sur image.

Mais notre travail va montrer tout l'enjeu de la complexité visibilité/lisibilité. Ainsi, Alain Mons nous invite à considérer l'affiche comme un mode symbolique d'une ville seconde, en revisitant ce que serait la ville dans sa réalité. Elle modifie l'agencement de l'espace par l'imaginaire institué de la réception instantanée. La réception instantanée désignant les effets directs et l'impact majeur de l'affichage urbain sur le consommateur de la matière publicitaire. Dès lors, l'affiche est considérée comme un élément de connaissance de la ville, un marqueur territorial. On peut ainsi, à travers ses affiches, apprendre sur un lieu et sur sa place dans la ville.

De plus, l'affiche constitue la matérialité de la ville et met à jour sa double fonction d'espace économique (affiche publicitaire) et d'espace de pouvoir (affiche politique ou institutionnelle). Or, les études de type sémiotique en matière d'affichage ont jusqu'à présent porté presque exclusivement sur l'affiche elle-même, sur ses genres, sur ses thématiques et sur sa composition interne. C'est d'ailleurs ce que Jacques Fontanille, appartenant au courant de la sémiotique française nomme «icono-texte ${ }^{2} »$. On peut donc penser que la sémiotique s'est jusqu'alors intéressée davantage à l'icono-texte de l'affichage urbain qu'à la complexité de celui-ci.

La sémiotique du discours fait une différence entre les enjeux situationnels et le contexte, c'est-à-dire la co-présence de caractéristiques qui accompagnent l'affiche. Et c'est dans cette perspective que nous nous plaçons pour étudier notre objet.

D'après Jean Didier-Urbain, le contenu du message est exposé aux aléas du contexte. "Des paramètres situationnels altèrent la réception du message, détournant ou infléchissant, pour le meilleur comme pour le pire, l'information énoncée. Selon les circonstances, la situation, en enrichissant ou, au contraire, en fragilisant le contenu de l'affiche et

\footnotetext{
${ }^{1}$ Alain Mons, L'affiche et la ville, les métamorphoses entrelacées, in Dans la ville, l'affiche, Revue EIDOS, n²4, Tours, 1993.

2 Jacques Fontanille, Affichages : de la sémiotique des objets à la sémiotique des situations, in Revista dell'Associazionze di Studi Semiotici on-line, p.1.
} 
Un nouveau regard sur l'affichage urbain...

sa rhétorique, a valeur d'adjuvant ou d'opposant dans le procès de communication. ${ }^{3} \gg$

Ainsi, pour Jean Didier-Urbain, il faut penser l'affiche dans ses liens à l'environnement dans lequel elle s'intègre (détermination spatiotemporelle : style du lieu) et dans sa transformation des relations entre les habitants et cet environnement. Toutefois, ces différents points de vue dénotent d'une réelle complexité lorsqu'il s'agit d'aborder la signification de l'affichage en général.

Pour notre part, nous allons aborder la signification de l'affichage au travers de l'analyse de discours. Cependant, il semblerait que les pratiques analytiques de discours restent souvent insuffisantes lorsqu'il s'agit de rendre compte de cet objet qu'est l'affichage urbain. La difficulté réside dans la capacité à lier la dimension matérielle (on évoque ici les notions de supports de l'affichage ainsi que leur disposition) de cet objet à sa complexité significative. Nous entendons par dimension significative à la fois le contenu élargi aux supports mais aussi la « nature » de l'impact sur le destinataire.

C'est à partir de cette difficulté que nous avons dessiné notre orientation méthodologique car il nous a semblé pertinent, dans l'étude que nous proposons, de tenter de combiner la dimension matérielle et la dimension significative de l'affichage. Dès lors, nous nous sommes placés dans ce qu'il est convenu d'appeler la sémiotique des objets.

Notre démarche consiste à proposer l'analyse d'un affichage urbain dans un lieu particulier; le passage Saint Rémi. A partir d'un protocole d'analyse que nous avons élaboré à la lecture des travaux de Jacques Fontanille ${ }^{4}$, nous allons présenter les résultats de nos observations sur l'hétérogénéité et la diversité du lieu afin d'émettre une hypothèse sur quelques aspects de la fonction signifiante et sur l'interaction communicationnelle avec les passants. Notre raisonnement est articulé autour de cette volonté de dépasser le rapport entre le texte et le contexte pour étudier les enjeux situationnels. Nous avons donc adopté une démarche exploratoire et expérimentale.

\footnotetext{
${ }^{3}$ Jean Didier Urbain, Eléments de pragmaffiche, in Dans la ville, l'affiche, Revue EIDOS, n4, Tours, 1993, p.15

${ }^{4}$ Jacques Fontanille, Affichages: de la sémiotique des objets à la sémiotique des situations, in Revista dell'Associazionze di Studi Semiotici on-line.

Alexandre Zinna et Jacques Fontanille, Les objets au quotidien, NAS, Editions Pulim, 2005.
} 
Analyses

Le postulat sémiotique actuel est de considérer que tout discours visuel avec ou sans support participe d'une forme de vie à partir de ce qu'il est convenu d'appeler la situation sémiotique. Nous replaçons l'aspect conceptuel et définitionnel, en question ici, avant d'en venir à l'analyse proprement dite.

Cette approche nous a paru intéressante pour notre objet d'étude car elle maintient le lien entre les objets matériels et leurs supports. Ce travail dépasse l'aspect strictement pragmatique et informationnel de l'affichage, sans le minimiser pour autant, pour se concentrer sur une relation spécifique, une relation tensive entre la lisibilité et la visibilité des affiches. Nous avons choisi pour lieu, la galerie bordelaise. Pour cela, nous avons réalisé, en quelque sorte, une enquête de terrain, une observation en situation, pour mettre en perspective une réflexion sur l'opérabilité de la sémiotique et de la situation sémiotique dans le cadre de l'affichage urbain. Dans notre démarche empirico-déductive, nous avons également construit un questionnaire, mais qui n'a eu toutefois qu'une valeur adjuvante à notre protocole.

Quand l'affichage du passage St Rémi rencontre le protocole de Jacques Fontanille

\section{Présentation du Passage Saint Rémi}

Nous avons choisi de réaliser notre étude de terrain dans la galerie bordelaise du centre ville, située au carrefour entre la rue Sainte Catherine et la rue St Rémi ${ }^{5}$, pour diverses raisons. Tout d'abord, ce lieu nous semblait complexe par la multiplicité de ses supports d'affichage. De plus, la difficulté du lieu nous paraissait être un challenge pour amener de nouvelles pistes de réflexion aux nombreux travaux déjà réalisés sur l'affichage urbain.

L'emplacement du passage Saint Rémi semble stratégique : c'est un carrefour entre les magasins, la place de la Victoire, le Grand Théâtre. Le lieu impose donc une fonctionnalité utilitaire ou pragmatique : les commerces et/ou l'information culturelle. Ensuite, c'est une galerie marchande ; elle a donc déjà une fonction de manipulation, de « fairefaire » sur les passants. Les vitrines peuvent ainsi arrêter les passants mais cette galerie est aussi et surtout un lieu de passage entre deux lieux stratégiques de la ville et un abri. De plus, la diversité des commerces est grande. Ici l'on pourra tout à la fois réserver une place pour un prochain concert, acheter de la lingerie féminine, trouver un

\footnotetext{
${ }^{5}$ Illustrations photographiques en annexe de l'étude, p.26.
} 
personnage de $\mathrm{BD}$ en $3 \mathrm{D}$ dans un magasin de jouets ou prendre un café et se restaurer.

Par définition, la galerie constitue deux prédicats :

- Une action déambulatoire proposant un passage entre deux rues de la ville.

- Une action marchande.

Elle comporte également deux fonctions :

- C'est un mode de raccourci.

- Ce lieu a une fonction pragmatique : il permet de se protéger du froid ou de s'abriter de la pluie.

La galerie est un espace à la fois ouvert, grâce à une grande verrière et à ses deux larges entrées, et fermé. Elle propose aux passants trois parcours possibles : les bordures (droite et gauche) et l'allée centrale. Ses vitrines, ses affiches sont implantées un peu partout provoquant une confrontation entre l'affichage légitime et sauvage. Son architecture est ancienne et esthétique. Il semble alors intéressant de nous arrêter quelques instants sur le caractère architectural de ce lieu. La Galerie Bordelaise fait partie intégrante du paysage bordelais depuis plus de 170 ans. Elle est d'ailleurs classée depuis le 29 octobre 1975 à l'inventaire supplémentaire des bâtiments de France. La construction de ce bâtiment date de 1834. Première Galerie de ce type en Europe au début du XIX ${ }^{\mathrm{e}}$ siècle, elle a pour originalité d'avoir construit un décor, composé de sculptures, de marbres et de bronzes, de dorures et de glaces. On note que la corne d'abondance ci-dessous est n symbole récurrent de la galerie :

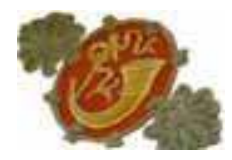

Deux portiques formés d'une voûte plein cintre, soutenue par des colonnes de marbre ouvrent et ferment la galerie. On peut remarquer encore le caractère élégant, grandiose de l'édifice. La richesse de sa décoration est reflétée par la multitude des glaces qui la jalonnent. $\mathrm{La}$ spécialité du magasin est inscrite en lettres d'or sur un fond orangé audessus de chaque vitrine auquel est accolé le nom de la profession. Contrairement à la plupart des galeries marchandes, on trouve des 
Analyses

appartements au-dessus des magasins. ${ }^{6} \mathrm{~A}$ cette première présentation de la Galerie Bordelaise, nous pouvons ajouter que l'on observe devant chaque magasin, des drapeaux, pour signaler les différentes enseignes. De plus, au milieu de la galerie, on voit des supports autonomes permanents (panneaux en bois à deux faces, peints, de type publicitaires comme celui du magasin de photographie) ou carrément des supports cubiques avec des affiches placées sous des vitrines en plastique (comme celui du box-office).

En fond sonore, on entend une musique classique toujours présente mais à faible niveau. Le lieu est un lieu de discontinuité crée par les différents territoires des magasins mais sa continuité est assurée par le lieu lui-même ou pour être plus précis par son architecture et sa verrière. Ainsi, on peut constater que ce lieu de passage est le théâtre d'un dialogue muet entre son architecture, sa fonctionnalité, et son affichage (support). Parce qu'il est un lieu de passage, ce lieu comporte des contraintes. Et cette dimension de contrainte nous a particulièrement intéressée. En effet, nous pouvons dire que ce lieu de passage ressemble à un tunnel dans lequel s'enchaîne une succession de vitrines des différentes boutiques jusqu'à donner l'impression qu'elles se télescopent.

Enfin, nous avons pu remarquer, outre les vitrines de magasin, la présence de panonceaux définissant ce qu'il est convenu d'appeler un affichage légitime ou institutionnel, qui cohabitent avec un certain nombre de spots dédiés à l'affichage qualifié de sauvage (affiches de spectacles ou autocollants). Or, c'est justement sur cette confrontation là que porte notre étude. Ce lieu va nous permettre d'envisager le rapport entre différents types d'affichages urbains et les enjeux situationnels qu'ils mettent en place, à travers la convocation de deux concepts, ceux de lisibilité et visibilité, que nous reconsidérerons à l'aune de nos analyses.

\section{Quelques éléments de sémiotique : des postulats au protocole d'analyse}

D'emblée, précisons que nous sommes dans une visée théorique et méthodologique qui s'attache à appréhender la construction de la signification de l'objet d'analyse. Notre démarche s'inscrit dans la sémiotique de la situation définie par Jacques Fontanille et qui considère l'hétérogénéité de l'objet dans la production et la réception

\footnotetext{
${ }^{6}$ Nous avons trouvé ces informations sur le site de l'Association de la Galerie Bordelaise : $\underline{\text { http://www.galerie-bordelaise.com/histoire.htm. }}$.
} 
de significations. Ainsi, on opère au plan méthodologique un déplacement de la sémiotique des énoncés vers la sémiotique de l'objet et de la situation.

Le caractère situationnel est débattu depuis longtemps en sémiotique. Il est opportun de retracer l'évolution de la sémiotique sur ce point. L'alternative sémiotique est celle de l'ontologie ou de l'anthropologie : depuis Platon, on a toujours privilégié l'être du sujet, son âme et la dimension ontologique du sens au lieu de ses pratiques. François Rastier ${ }^{7}$ précise que, depuis les Lumières, le cerveau aurait remplacé l'âme, mais que la raison resterait le fondement de la sémiotique. C'est pourquoi, en sémiotique, la signification est abordée sur deux dimensions: une première dimension serait topologique, celle des unités discrètes ou dites discontinues. Le discours est alors, pour résumer, la mise en oeuvre d'un système, citons par exemple l'analyse de l'organisation narrative d'un texte-énoncé qui se traduira par la jonction de sujets et d'objets dans un jeu de perte et de gain. Prenons un parangon de la grammaire textuelle du discours publicitaire : « avec la crème $\mathrm{K}$, luttez contre les effets du temps ». La crème est un actant sujet opérateur d'une programmation prédiquée sur le mode de la lutte afin qu'un sujet (le lecteur cible) ne soit pas conjoint à l'objet de valeur "vieillesse ». Une deuxième dimension serait dite énergétique. Cette conception de la signification impose de fait un amont et un aval : pré conditions de la signification (dimension énergétique ou continue), en amont de la signification linguistique et dimension topologique (linguistique ou discontinue) se situant alors en aval.

Selon la sémiotique, le discours s'appréhende sur le plan discontinu (notion de place, de structure, modélisé par la notion de prédication), et aussi sur ce que l'on nomme le « discours en acte ». Le discours dit « en acte » relève de ce qui est l'émergence de la signification, de ce qui fait advenir la signification dans un discours. Pour dire les choses autrement, notre démarche s'appuie sur une question épistémologique, propre à la sémiotique: Qu'y a-t-il derrière les structures sémionarratives, qu'y a $t$ il derrière les programmations discursives (Qui fait quoi ? Pourquoi ? Dans quel but ?). Ce questionnement est sensible depuis "Sémantique Structurale» de Algiras Julien Greimas, qui définissait justement la sémiosis (plan de l'expression /plan du contenu) comme une dyade articulant une dimension extéroceptive (sémiotique d'un monde naturel) et une dimension intéroceptive (de

${ }^{7}$ François Rastier, L'action et le sens pour une sémiotique des cultures, Journal des anthropologies $\mathrm{n}^{\circ} 85-86,2001$. 
Analyses

l'ordre du symbolique, du signe). La dimension extéroceptive du discours se situe au plan de ses effets sensibles, du ressenti même. La deuxième composante de cette dyade, la dimension intéroceptive touche, elle, l'organisation strictement linguistique du discours.

En outre, cette approche de la signification s'appuie également sur un arrière-plan phénoménologique. En effet, forte des positions du phénoménologue Maurice Merleau-Ponty, qui avançant que le perceptif et le sensible sont tous deux porteurs de signification. La signification serait alors un rapport entre discontinu [extéroceptif] et continu [intéroceptif]. Toujours en accord avec la phénoménologie, la sémiotique utilisera une instance spécifique, celle du corps pour médiatiser ces deux dimensions, pour envisager autrement la sémiosis. Dans ce cadre, apparaît le corps comme instance spécifique au discours : la proprioceptivité. Elle permet de relier le monde naturel non signifiable et le monde symbolique (par exemple, la langue). Cette position abstraite du corps, dit proprioceptif, est quelque fois qualifié d'imaginaire sans qu'on s'interroge réellement sur le sens à donner à ce terme. Nous soulignons que le primat du corps comme instance énonciative ne définit le corps ni comme un ensemble d'organes ni comme un corps imaginaire ou un corps pulsionnel mais plutôt comme un corps phénoménologique, instance sensible de perception. Il est une médiation entre monde naturel et monde linguistique. Pour reprendre les termes de Maurice Merleau-Ponty, ce corps n'est pas un «je pense » mais un « je peux ». Il est appareillé perceptivement.

Nous verrons que cette étude nous permet d'interroger l'instance du corps défini sous l'angle phénoménologique. Nous émettons la possibilité de lire sous ce choix méthodologique la construction d'un fantasme inhérent à la sémiotique. Il relèverait d'un imaginaire qui considèrerait que l'on puisse opérer une saisie originelle du sens. Quelques propos de Jacques Fontanille sur le sens peuvent être ainsi entendus ; le sens, dit-il, serait ce lieu « où se joue et se rejoue sans scène la scène primitive de la signification c'est-à-dire l'émergence du sens à partir du sensible. ${ }^{8} \gg$

Pour mener cette étude, nous suivons en premier lieu ce postulat sémiotique. Afin de rendre compte de la sémiosis de l'affichage urbain, la sémiotique prend comme point d'insertion l'instance corporelle, soit le corps médian entre monde des choses et monde intelligible. Cette attitude analytique nous permet de mieux prendre en

\footnotetext{
${ }^{8}$ Jacques Fontanille, Sémiotique du discours, PULIM 1999, p 39.
} 
compte notre objet d'étude, c'est-à-dire, l'interaction de l'objet (affiches du passage St Rémi) avec les passants de la galerie.

De plus, nous travaillons sur la dimension objectale de la signification. Nous précisons dès maintenant ce que cela veut dire au plan méthodologique.

La notion d'objet est d'abord considérée dans la topologie discontinu/continu dans la théorie sémiotique. Dès qu'on s'attache à définir la notion d'objet, on s'éloigne de sa détermination sémantique et définitionnelle au profit de la spécification de ses propriétés. Alexandro Zinna, spécialiste en sémiotique des objets ${ }^{9}$, souligne cet état de forte polarisation fonctionnelle de la signification, dès lors qu'elle s'ouvre à sa dimension objectale. L'objet est défini en sémiotique comme relevant «des structures plus ou moins matérielles dotées d'une morphologie, d'une fonctionnalité et d'une forme identifiable dont l'ensemble est destiné à un usage ou une pratique plus ou moins spécialisées et qui pour certains sont dotés d'une surface d'inscription pour les textes-énoncés..$^{10} \gg$ Un simple exemple de l'auteur, la lettre, illustre la redéfinition de l'objet en sémiotique. Il en dégage aisément deux pratiques évidentes : celle de l'épistolaire et celle de la communication et de la circulation des objets dans la société.

On peut dire que l'objet est saisi soit sur une version intense (objet comme support -inscription/empreinte) soit sur une version extense (objet comme acteur dans une pratique prédicative, par exemple manipulatrice). Ainsi la notion de - pratique - serait un niveau de pertinence particulier entre le monde des objets à proprement parler et la situation plus générale dans laquelle l'objet s'inscrirait. Nous avons relevé ci-dessus un premier niveau de pertinence : le texte-énoncé puis un deuxième, celui de la pratique. Il faut à présent, en considérer un troisième ; celui de la situation sémiotique.

Tout d'abord, il est important d'insister sur la disjonction définitionnelle entre situation et contexte. La sémiotique définit la situation autrement que par ce qui environnerait le texte. Il s'agit d'une configuration d'intégration des deux niveaux précédents qui lui «rassemblerait tous les éléments nécessaires à la production et à

\footnotetext{
${ }^{9}$ Voir Jacques Fontanille, Les objets au quotidien, PULIM, 2005.

${ }^{10}$ Ibid, p. 196.
} 
Analyses

l'interprétation de la signification d'une interaction communicationnelle $»^{11}$.

Ce terme de « situation » est apparu dès les années 80 dans les travaux d'un autre sémioticien; Eric Landowski. Il proposait jusqu'alors de rendre compte de la sémiotisation du contexte, c'est-à-dire des variables, des surdéterminations du contexte, que représentaient le pragmatique et l'empirique des interactions sociales. Il s'appuyait essentiellement sur l'armature modale qui mobilisait, à cette époque, la recherche théorique et ses applications au plan de l'analyse de discours. Aujourd'hui, la sémiotique propose d'y agréger d'autres éléments en définissant deux modes macro-sémiotiques à partir de l'articulation tensive de la "situation scène» et de la "situation stratégique », autrement dit en cernant articulation tensive qui existe entre lisibilité et visibilité d'un objet signifiant. Nous revenons maintenant sur ces concepts en nous penchant sur la manière dont la sémiotique considère l'objet affiche.

\section{L'affichage à partir de la sémiotique des objets}

Nous insistons sur le fait que l'enjeu est de dépasser une analyse cantonnée aux unités verbales/iconiques à leurs valeurs dénotatives/connotatives auxquelles on adjoint leurs éléments plastiques. A ces fins, nous avons construit un "protocole », à partir des données exposées préalablement ainsi qu'à partir des travaux de Jacques Fontanille sur l'affichage. Pour en résumer la teneur nous avons retenu la définition de la situation sémiotique selon deux dimensions :

\section{La situation scène}

$\mathrm{Au}$ plan du discontinu, du linguistique, Jacques Fontanille parle de " petite scène " pour qualifier le mode prédicatif qui la caractérise. Cette dimension vise à rendre compte des modes programmatifs que mettent en action l'affiche. On peut ainsi appréhender la manière dont chaque affiche ou type d'affiches s'ajuste à son espace d'inscription. Très simplement, on détermine à la fois les rôles, les actes, les modalisations ainsi que le type de dispositif énonciatif mobilisés par l'affichage.

\section{La scène stratégique}

Elle convoque la dimension sensible du discours de l'affiche, l'intéroceptivité, c'est pourquoi nous l'abordons aux planx figuratif et

\footnotetext{
${ }^{11}$ Jacques Fontanille, Les objets au quotidien, PULIM, 2005.
} 
thématique, mais aussi isotopique en tentant de cerner le mode d'interaction avec le passant.

Bien entendu ces deux dimensions ne s'opposent pas, elles s'inscrivent dans une tension entre programmation et stratégie.

Nous exposons ici l'enjeu prédicatif: Quel mode d'action existe-t-il entre l'affichage et l'observateur? A ce niveau là, c'est la lisibilité de l'affiche qui est en jeu. Que permet-elle ? Oblige-t-elle à faire quelque chose ? Par exemple, a-t-elle uniquement une visée promissive sans prédication actionnelle actualisée, ou impose-t-elle un acte performatif au passant ? La scène stratégique instaure, elle, la dimension de la visibilité. En effet, par la scène stratégique, on tente d'approcher les modes d'appropriation visuels convoqués par l'affichage. Dans notre étude, nous repérons le rapport qui s'instaure entre le passant et les différents types d'affiches. C'est par le repérage éventuel du degré de contamination perceptive ou sémique entre affichage dit institutionnel et sauvage, médiatisé par l'instance perceptive de l'œil, du corps sensible du sujet (le passant), que nous lisons le rapport entre lisible et visible. Ce rapport se construit à travers la dualité des deux scènes, et plus particulièrement de leur tension prise par le jeu d'une certaine manipulation. Cela veut dire qu'il y a une confrontation entre une manipulation dite restreinte, qui vise à la qualification prédicative d'un observateur en actualisant la dimension lisible de l'affiche, et une manipulation dite stratégique, sorte de $"$ réglage tactique ${ }^{12} »$. Ce réglage tactique instaure une potentialité de captation de l'observateur par l'affiche en soi en saisissant le passant dans sa présence même.

Nous allons maintenant vous présenter les résultats de notre analyse à travers cette partition lisible - situation scène et visible - situation stratégique, en synthétisant nos résultats sur les trois emplacements qui ont retenus notre attention. Nous avons pu ainsi poser la problématique suivante :

\section{Problématique}

D'un point de vue sémiotique, l'affichage est soumis à des contraintes de programmation et à des contraintes stratégiques qui définissent la dimension situationnelle de sa signification, qui appréhende à la fois l'hétérogénéité et la complexité de l'affichage de ce passage Saint Rémi. Nous déterminons le type d'articulations qui relie ces deux contraintes.

\footnotetext{
${ }^{12}$ Terme emprunté à Jacques Fontanille dans Les objets au quotidien, PULIM, 2005.
} 
Analyses

\section{Hypothèses}

Quel est le type d'articulation ${ }^{13}$, de tension que ce lieu génère ? Y'a-til concurrence entre les dimensions ? Quel est le rapport entre lisibilité et visibilité des divers modes d'affichage? Quel est le jeu entre lisibilité et visibilité des divers modes d'affichage?

\section{Synthèse de l'étude sémiotique du passage St Rémi}

A partir de nos premières remarques, nous avons pu observer trois niveaux d'embrayage différents dans le passage Saint Rémi, qui sont : les vitrines des commerçants, un emplacement dédié à l'affichage sauvage et les affiches d'un «méta-énonciateur». Notre lecture de l'analyse se base sur l'établissement du protocole qui va se décomposer entre situation-scène (lisibilité) et situation stratégique (visibilité).

\section{L'enjeu de lisibilité du Passage St Rémi}

La situation prédicative engage une relation entre le contenu de l'affiche et l'observateur mais elle prend aussi en compte la nature et le rôle des objets supports. Nous avons pour chaque affiche cherché à dégager le rapport entre l'observateur et la prédication présupposée par l'affiche. Autrement dit, nous avons voulu savoir ce qui dans l'affiche est un acte promissif d'action. Le second point qui nous paraissait important était de savoir s'il existait des actes de qualification identifiables: l'affichage est-il commercial, institutionnel... et si ces qualifications étaient d'ordre occasionnel ou permanent? Enfin nous nous sommes penchés sur les supports eux mêmes afin de dégager leur rôle comme actant à part entière dans une situation d'affichage.

\section{Emplacement 1 : Les affiches à l'entrée de la galerie}

Six affiches sont disposées à l'entrée de la galerie. Ici, les affiches sont de type commercial à visée culturelle (concert, soirée thématique, exposition) et prennent appui sur un support autonome autrement dit débrayé. Ce sont les illustrations (couleur, photographie) qui instaurent clairement un acte de lisibilité du contenu.

Arrêtons-nous sur les notions d'espace de réalisation et d'affichage embrayé. Jacques Fontanille distingue deux sortes d'objets-supports,

\footnotetext{
${ }^{13}$ Par articulation, nous entendons un jeu tensif, un rapport de force entre les deux scènes. L'analyse de ce jeu tensif dans le passage St Rémi nous permettra d'orienter la réflexion sur l'affichage urbain vers de nouvelles ouvertures.
} 
ceux qui fonctionnent comme interfaces embrayées et ceux qui fonctionnent comme supports autonomes. Lorsque l'espace de lecture et celui de la réalisation sont concomitants il s'agit d'une interface embrayée telle une vitrine proposant des produits que l'on trouve à l'intérieur même du lieu d'affichage. Le support autonome est débrayé quand il n'entretient plus de lien perceptible avec le lieu de réalisation tel un panneau mural. Dans le premier cas, "la proximité entre les deux phases et les deux espaces est un adjuvant puissant pour l'efficacité de la proposition, dont toutes les conditions sont alors réunies à l'intérieur de la seule situation-scène ${ }^{14}{ }^{\prime}$.

Dans le second cas, l'objet est simple support de proposition et de promesse "qui laisse à l'affiche et à la situation stratégie la charge de régler le passage à l'acte et l'efficacité du dispositif $f^{15} »$. C'est dans ce cas en particulier qu'il est d'autant plus nécessaire de considérer la situation stratégique de l'affichage.

A proximité de ces affiches, sur le même mur, figure un pannonceauaffiche rouge et en plastique. La couleur de cette affiche est très visible ; elle se détache de la typographie. L'affiche-panneau est aussi permanente et très contextualisée puisque le lieu de réalisation se situe non seulement dans la galerie mais à l'emplacement exact de l'affichage. La qualification est ici aussi préventive.

Quelques pas après l'entrée proprement dite de la galerie figurent deux affiches identiques placées sur deux colonnes de part et d'autre d'une marche. Elles mettent en garde le passant contre une éventuelle chute. L'injonction "attention à la marche », véritable performatif est tout à fait lisible. Elle est disposée à deux reprises de part et d'autre de la marche. Mais c'est le pictogramme (flèche indicatrice plus que la typographie) qui attire le regard par sa simplicité et ses couleurs rouge et noir (qui se détachent de façon évidente). C'est un affichage dit permanent et embrayé puisqu'il est énoncé par la galerie.

\section{Emplacement 2 : Le magasin de photographie}

Nous pouvons faire plusieurs remarques générales concernant ces affiches. Premièrement, nous pourrions dire qu'il ne s'agit pas d'affichage sauvage anarchique, dans la mesure où il n'y a pas de chevauchement brutal et systématique avec les autres affiches du moment.

\footnotetext{
${ }^{14}$ Jacques Fontanille, Affichages : De la sémiotique des objets à la sémiotique des situations, Rivista d'ell Assozione Italiana di Studi, Semiotoca on-line, p13

${ }^{15}$ Ibid.
} 
Analyses

II en est de même pour toutes les affiches. Ce point ne sera pas détaillé pour chacune d'entre elles. On parle ici d'affichage débrayé autonome. Nous avons aussi remarqué que pour toutes les affiches à une exception près, l'acte de qualification est commercial à portée culturelle (théâtre, musique exposition) et qu'elles ont toutes un caractère occasionnel.

En ce qui concerne le support, c'est apparemment une ancienne vitrine à l'abandon appartenant au magasin de photographie qui jouxte l'espace d'affichage étudié. C'est une vitrine «descendante » ${ }^{16}$ qui a été investie par un affichage débrayé. Il existe diverses sortes de vitrine : les vitrines actives (commerce actif) et les vitrines inactives, ascendantes (en cours d'aménagement) ou descendantes (en cours de vente ou de transfert). Dans chacun des cas, la lecture de la situation sémiotique diffère, il est donc important de prendre en compte le statut de chaque objet-support. Jacques Fontanille insiste d'ailleurs à ce propos sur l'importance de bien différencier l'environnement de l'affichage qui relève de la situation matérielle (le contexte seul) et la situation sémiotique qui elle, relève de la situation formelle qui en d'autres termes unifie scène et stratégie énonciatives.

La lecture des affiches semble s'appuyer sur des formants visuels de couleur plutôt que sur la typographie. Ce sont les illustrations qui, sur la plupart des affiches, semblent prégnantes au niveau du lisible. Il est à noter que pour la compréhension de certaines de ses affiches, l'observateur doit se transformer en lecteur afin d'aller piocher une information spécifique. S'il ne reste qu'observateur, ce sera par déduction qu'il pourra inférer la nature de la proposition. Nous pourrions dire qu'il y aurait une connivence entre l'affiche et l'observateur.

Prenons par exemple les affiches du «Krakatoa», "Fleur de Fougère » ou même encore «Le Festin ». Ce n'est dans les deux premiers cas que parce qu'elles ressemblent à un type d'affiche particulier que l'observateur peut supposer qu'il s'agit de proposition de type programmation de concert («Krakatoa») ou exposition («Fleur de Fougère »). La connivence pour « Le Festin» se place sur un autre plan: l'affiche fait appel à une culture partagée, si l'observateur ne connaît pas la revue bordelaise il ne peut savoir qu'il s'agit là de la présentation d'un nouveau numéro à sortir.

\footnotetext{
${ }^{16}$ Terminologie empruntée à Jacques Fontanille, Affichages : De la sémiotique des objets à la sémiotique des situations, Revista i dell Associazione di Studi Semiotica on-line...
} 
On note aussi la présence d'un seul empiètement d'une affiche sur une autre. Il semblerait que l'initiative émane d'un particulier ou du moins d'une structure moins importante que les institutions culturelles qui proposent les autres concerts et expositions. La portée de l'affiche est d'ailleurs de nature différente puisqu'elle fait figure de petite annonce.

Enfin, il est à noter la particularité de l'affiche «Root'System » dont l'accroche visuelle se fait surtout par le sens de l'affichage. C'est en effet la seule affiche qui se présente de façon horizontale.

\section{Emplacement 3 : la vitrine de la pharmacie}

Sur la vitrine d'une pharmacie, sont disposées depuis l'intérieur, des affiches représentant des produits de parapharmacie, accompagnées de leur prix. De prime abord, on observe que les prix des produits sont les plus lisibles. Le produit quant à lui n'est pas clairement identifiable en raison de la mauvaise qualité de l'impression de l'affiche, qui semble avoir été faite par le commerçant lui-même. Pour ces affiches, il s'agit d'un acte de qualification d'ordre commercial.

Cet affichage est sans doute occasionnel car il est placardé sur la vitrine telles que le sont généralement les promotions temporaires. Cependant aucune date n'indique qu'il s'agisse d'une initiative promotionnelle particulière. C'est donc une situation éminemment contextualisée, dans la mesure où l'espace de réalisation se trouve sur le lieu d'affichage. Le mode d'affichage est embrayé sans pour autant qu'on puisse le qualifier de sauvage puisque il apparait même très « rangé » avec un effet de répétition. La vitrine est ici dite « active ».

\section{L'autocollant}

Cette affiche est peu lisible de loin. On distingue seulement deux couleurs : noir et blanc, avec vraisemblablement un personnage qui sourit. Le texte qui accompagne l'illustration n'est lisible qu'une fois que l'on s'approche de l'autocollant dont la dimension est assez réduite par rapport à une affiche courante. La qualification est difficile à cerner voire très incertaine. Nous pouvons cependant dire qu'elle n'est pas commerciale. Elle pourrait être le résultat d'une initiative commune de la part des commerçants de la galerie. Le «slogan» accompagnant le dessin étant le suivant « Galerie Bordelaise. Dingue de foot $»$. Il semblerait en effet que la galerie soit à l'initiative de cet affichage. Ce pourrait être en quelque sorte un affichage d'opinion ou de position, qui est en clair décalage avec toutes les autres affiches. Un objet «symbolique » qui réunit tous les commerçants. L'affiche, par son intemporalité, son propos et sa nature (autocollant) semble appeler à rester en place en permanence. 
Analyses

L'emplacement de l'objet est ici particulier. L'affiche est collée sur un tuyau d'évacuation des eaux. Le support est autonome par rapport à chaque vitrine, dans sa totalité, il s'agirait sans doute alors d'un support embrayé. Ce support atypique soulève la question des «propriétés de pertinence » des objets-supports. Un tuyau de descente des eaux de pluie est assez inattendu comme support d'affichage et « $n$ 'est pas supposé faire partie des éléments pertinents de la situation d'affichage $\gg{ }^{17}$. Pourtant il n'est pas rare de voir ces objets devenir des supports d'affiche. Jacques Fontanille écrit alors que "leur opérativité $n$ 'est pas moindre que celle des affichages officiels $\gg{ }^{18}$

\section{Conclusion}

Les trois spots ont des niveaux d'interface très différents. La lisibilité est fortement contrainte par la nature matérielle du support. La vitrine de la pharmacie ne demande pas à «être déchiffrée » si l'on puit dire. La manipulation restreinte de l'entrée de la galerie dans l'univocité de sa prédication performative ne laisse guère d'ambiguïté de lisibilité. L'emplacement d'affichage sauvage semble alors entrer en conflit, et cela d'autant plus qu'il s'inscrit dans un cadre de manipulation restreinte et de lisibilité forte. Par ailleurs, il laisse émerger une présence sensible d'ordre perceptive, la qualification demeure, certes possible, mais plurivoque comme, l'indique les réponses des passants interrogés à partir de notre questionnaire («Pas vraiment de visibilité de l'affiche ", "L'affiche ne m'a pas interpellée », "Il y a certes un aspect visuel, mais l'affiche ne m'a pas interpellée », "Je ne me souviens pas des supports ».). Nous avons dégagé de manière synthétique l'ensemble des propriétés thématiques et plastiques des affiches.

\section{Emplacement 1 : Les affiches de l'entrée de la galerie}

Selon l'axe de déambulation, la visibilité change : l'affiche est visible lorsqu'on sort de la galerie, presque invisible lorsque l'on y entre.

\section{$\diamond$ Disposition}

Les affiches sont scotchées les unes en dessous des autres, en fonction de leur taille et format. Elles sont peu nombreuses. On note un petit jeu sur les couleurs pour mettre en valeur les affiches ainsi qu'un jeu sur le mouvement interne des affiches qui entraîne un parcours visuel du regard sur les affiches.

\footnotetext{
${ }^{17}$ Jacques Fontanille, Affichages : de la sémiotique des objets à la sémiotique des situations, in Revista dell'Associazione di Studi Semiotici on-line p.7

${ }^{18}$ Ibid.
} 
Un nouveau regard sur l'affichage urbain...

\section{$\diamond$ Contraintes - Visibilité}

Si les textes sont assez lisibles de loin (taille des polices des gros titres), la faible exposition du mur à cet endroit (contraste avec la luminosité en sortant) diminue leur visibilité. Par effet de contamination aussi, elles forment un bloc d'affichage culturel évènementiel, à l'exception du panneau de prescription rouge au bas du mur, qui, étant fixe et permanent, constitue, avec les deux affiches sur les poteaux, la voix du méta-énonciateur de la galerie.

\section{$\diamond$ Prothèse de visibilité}

Le panneau en bois à deux faces du magasin de photographie est à considérer comme une prothèse, et devient donc un enjeu de visibilité. Cependant, la captation est imprévisible et suppose, nous semble-t-il, davantage un effet de glissement du regard chez les passants qui traversent le passage St Rémi. C'est une captation dont on mesure mal l'enjeu.

\section{Emplacement 2 : Les affiches du magasin de photographie}

\section{$\diamond$ Disposition}

Sur les panneaux muraux, les affiches forment un ensemble sans véritable relief. Elles sont disposées de manière très serrées, collées les unes contre les autres. L'une d'entre elles est même collée pardessus une autre. Formant un ensemble assez uniforme sur la gauche, les affiches invitent le passant à s'en approcher pour mieux les distinguer et les lire.

\section{$\diamond$ Contraintes :}

Les affiches se situent dans une zone déjà fortement saturée d'informations. Cette enfilade de vitrines de magasins, toutes de même hauteur et quasi identiques, crée une certaine uniformité. De plus, la surface disponible est réduite, ce qui constitue une contrainte supplémentaire pour attirer le regard du spectateur, puisque cet ensemble crée un bloc uniforme presque informe. Dans un espace " confiné », les affiches doivent trouver leur place dans la galerie. Un îlot est toutefois visible et forme une sorte de tache perceptive, kaléidoscope de formants de couleur, de bribes typographiques.

\section{$\diamond$ Isotopies :}

Le trait de sens commun de ces affiches relève de l'évènementiel, du culturel, marqué plus par un effet de contamination sémique que par une véritable catégorisation thématique lisible. Les affiches ne permettent pas un débrayage spécifique les unes par rapport aux 
Analyses

autres, même si on remarque un certain ajustement horizontal ou vertical des affiches les unes par rapport aux autres, en fonction de leur format. On note aussi quelques jeux de contrastes entre les couleurs des affiches pour les mettre en valeur.

\section{Emplacement 3 : Les affiches de la pharmacie}

\section{A / L'autocollant :}

\section{$\diamond$ Disposition :}

L'autocollant est isolé sur la canalisation et disposé en hauteur sur ce support atypique. Il attire l'attention par un jeu visuel sur le contraste entre le gris du support-objet et le noir et blanc l'affiche.

\section{$\diamond$ Contraintes :}

C'est un autocollant non promotionnel, sur un support inhabituel. Il semble décalé dans le jeu tensif. Mais le fait qu'il soit isolé en hauteur avec ce contraste de couleur, cette forme ronde et sur un support atypique, attire l'attention. C'est le support (le tuyau) qui le rend signifiant plus que significatif. Le détournement d'un objet comme prothèse d'affichage renforce la potentialité de visibilité et d'effets de captation.

- Le tuyau est un objet détourné, illégitime, qui crée une connivence avec le passant. Elle peut se lire comme une complicité dans sa proposition illicite, comme une astuce et une débrouillardise voire engagement militant. Toutes ces raisons participent à la légitimité des affichages standards. Nous sommes face à une renégociation du message par chaque lecteur, ce qui renforce la valeur culturelle et imposée des autres affiches, tout en dégageant l'originalité de cet autocollant. De plus, on relève une dimension ironique: l'affiche évoque quelque chose sur la relation de cette galerie avec la ville, sans le dire vraiment.

- Le tuyau révèle une «stratégie de visibilité ${ }^{19}$ » en « [monopolisant] la surface du tuyau, pour éviter toute tension ou concurrence avec d'autres propositions voisines. ${ }^{20} \gg$ On note alors une tension entre l'objectif informatif et l'objectif de captation esthétique. On se souvient davantage de l'originalité de l'affichage que du message. L'affichage officiel constitué par les

\footnotetext{
${ }^{19}$ Jacques Fontanille Affichages : de la sémiotique des objets à la sémiotique des situations, in Revista dell'Associazionze di Studi Semiotici on-line p.7.

${ }^{20}$ Ibid
} 
affiches institutionnelles (évènementiel) est au contraire bien réparti dans la galerie et accroche le regard du passant.

\section{$B$ / Les affiches de la pharmacie}

\section{$\diamond$ Disposition - Stratégie de visibilité :}

Les affiches sur la vitrine de la pharmacie sont toutes du même format (A4) et composées de la même manière (un produit en gros plan avec son prix en gros au dessous) de sorte que les stratégies de visibilité et leur lisibilité sont fortes. Elles sont disposées de la même manière avec un intervalle identique entre chacune.

\section{$\diamond$ Isotopies :}

Par effet de contamination, les affiches constituent un bloc d'affichage commercial qui va de pair avec l'activité de vente à l'intérieur de la pharmacie. L'enjeu du visible est somme toute évident. Il est plus intéressant de s'arrêter sur le conflit de manipulation entre les différents spots, c'est-à-dire sur la matérialité propres du support. Le cheminement d'un passant [traverser la galerie] / [s'arrêter ou pas dans une boutique] conditionne fortement l'enjeu de visibilité. Le degré de contrainte de qualification spécifique aux spots (pharmacie, entrée...) ne laisse aux autres (panneau de bois, colonnes de la galerie...) qu'un acte de l'ordre de [«je [affiche] suis présente]. Le conflit de qualification invalide leur fonctionnalité informative potentielle.

\section{Premier pas conclusif :}

Tout d'abord, nous pouvons dire que le protocole établi à partir des données théoriques de la situation sémiotique permet de mettre en évidence bien des aspects significatifs de l'affichage à l'instar des travaux préexistants (notamment les considérations générales de Jacques Fontanille ${ }^{21}$. Nous avons pu mettre en évidence l'importance des supports et de leur inscription spatio-temporelle, et montrer la portée d'une analyse qui ne s'en tient pas uniquement à l'icono-texte. Nous avons déterminé le rapport existant entre le promissif/prédicatif du lisible de l'affichage propre à ce passage, retenons par exemple que la lisibilité et les actes de qualifications de la vitrine de la pharmacie sont à la fois plus contraintes et plus prescriptives que celle du panneau de bois près du magasin de photographies. Ce même panneau

\footnotetext{
${ }^{21}$ Jacques Fontanille, Affichages : de la sémiotique des objets à la sémiotique des situations, dans Revista dell'Associazionze di studi Semiotici, en ligne.
} 
Analyses

de bois sur lequel se centre grand nombre d'affiches sauvages, crée, comme nous l'avons dit une mouvance instable entre lisible et visible, au bénéfice du visible par l'impression de «tache» que ce spot compose. L'acte d'ostentation et de captation préfiguré par le détournement même de cet espace dessine un rapport à l'observateur qui construit un horizon de simple présence d'éléments figuratifs.

Si nous faisions une simple comparaison avec d'autre lieux de passage tel que le métro, lieu dans lequel justement la captation doit rester fugitive face aux affichages qui jalonnent le parcours des usagers du métro, et où la prédication de l'arrêt doit rester improbable en raison de l'importance de fonction du défilement du visible de l'affiche ; on remarque dans le passage St Rémi, l'accentuation de l'enjeu tensif entre manipulation restreinte (lisibilité) et manipulation stratégique (visibilité). En effet, la stase possible entre prédicat (s'arrêter et/ou entrer dans une boutique) est ici, inhérente au lieu.

Une première proposition conclusive serait donc d'entériner dans notre étude les considérations déjà largement établies autour des enjeux significatifs de l'affichage urbain, en ajoutant que ce passage renforce considérablement la concurrence dans la course qualifiante du passant.

\section{Ouverture réflexive : une impasse ?}

Notre premier pas conclusif pourrait alors laisser croire que nous avons appliqué une méthode sémiotique dont nous aurions essayé d'explorer l'opérationnabilité de ce qu'il est convenu d'appeler la sémiotique des objets à un environnement particulier. Et finalement que nous sommes parvenu à confirmer des intuitions que nous avions en tête, avant même de nous lancer dans cette étude.

L'enjeu premier de cette analyse est de dépasser l'exercice sémiotique appliqué aux affiches, de dépasser la saisie de leur signification. Nous avons vu que la prédication et la manipulation restreinte est à mettre en relation avec leur degré de visibilité. Cette construction qui confronte affichage institutionnel et affichage sauvage fait ressortir un mode ostentatoire. Ces remarques se sont constituées en partant de l'hypothèse d'un corps, médiation perceptive, entre le monde des choses et celui des objets et le monde construit (lisible).

C'est dans cette optique que nous poursuivons notre réflexion. Le corps phénoménologique médiatisant lisible et visible, pourrait, selon nous, être réenvisagé au jeu même de ce que nous avons qualifié d'oscillation : rapport du lisible et du visible. Cette instance corporelle traduite jusqu'ici à partir de sa dimension sensible et intelligible 
obture toute possibilité d'explorer cette oscillation, en l'appréhendant plus avant. Nous émettons alors l'idée qu'il y aurait une sorte de doublure à l'instance phénoménologique, un au-delà du visible que pointe justement l'oscillation. Nous la qualifions «d'avant-œil», terme que nous empruntons à la psychanalyse. En partant au seuil de notre travail du corps phénoménologique et symbolique, nous parvenons dès lors au corps dans ses modes de jouissance, et plus exactement au corps parlant. Cela nécessite de marquer un temps explicatif quant à la définition de ces termes : «corps parlant» et «jouissance $»^{22}$. Ils ouvrent chacun de vastes espaces définitionnels sujets à discussions multiples. Pour notre part, nous nous en tenons à l'enseignement de Jacques Lacan, en replaçant ces termes en deux moments cruciaux de son enseignement ${ }^{23}$.

Nous distinguons un premier moment: le temps où la relation de la parole, la relation intersubjective, communicationnelle caractérise la démarche psychanalytique. Ce sont, en effet les fonctions du langage, et la relation à l'Autre, qui construisent le lieu de toutes les déterminations du sujet. Du côté du sujet, on considère alors que le symptôme est un sens non révélé, refoulement d'un signifiant traumatique. Du côté de l'Autre, c'est la validation du sens et une reconnaissance du sujet par cet Autre. La dimension symbolique est donc point d'accès majeur à l'être du sujet.

C'est bien sûr, à grands traits, mais nous retraçons l'existence d'un deuxième moment dans l'enseignement lacanien qui pourrait se résumer ainsi : il y a un ravalement de la pensée et du langage qui lui est subordonné, et même du «prestigieux» inconscient à des semblants, c'est-à-dire de simples moyens pour tenter de pallier au rapport difficile, dysharmonique, que tout être humain entretien avec son corps, avec le corps sexué, et le corps de l'Autre. Une cause à cette dysharmonie : le fait que nous soyons "parlant» affecte notre corps par des "événements de parole». On dit alors que le réel du corps sexué s'accorde mal à l'Autre. Le corps est dit divisé par le langage. Il y a disjonction entre le corps qui n'est en liaison qu'avec lui-même (Jouissance Une) et l'Autre (lieu du langage, de la dimension symbolique). Notre imaginaire constitué de fantasmes et des menues objets de jouissances propres à chacun d'entre nous, tente

\footnotetext{
${ }^{22}$ Nous signalons que nos propos sont évidemment un raccourci succinct sur les différents paradigmes de la jouissance dans l'enseignement de Lacan

${ }^{23}$ Nous nous appuyons ici sur un article de J.A. Miller, Les Six paradigmes de la jouissance, $\mathrm{n}^{\circ} 43$ de « La Cause Freudienne ».
} 
Analyses

de nouer tout ça: nous avons un corps «a-perceptif», dénoué de l'Autre. Jacques Lacan invente un nouveau terme : le " parlêtre ».

Ainsi, l'oscillation du lisible et du visible, décelé dans notre analyse, nous invite à déplacer le curseur initialement situé sur la dimension symbolique et perceptive vers celle de la jouissance. La tension lisible /visible propre à ce lieu d'affichage urbain, par la doublure du "parlêtre » et de sa jouissance, nous permet de réinterroger la captation du passant. On sait par ailleurs que Maurice MerleauPonty $^{24}$ a défini cette fonction de captation. Il souligne à maintes reprises que "nous sommes des êtres regardés dans le spectacle du monde. » Cependant, ni la phénoménologie ni surtout la sémiotique n'ont pris la juste mesure des conséquences du "parlêtre » sur son rapport au monde, c'est-à-dire une alliance difficile, entre être un corps et avoir un corps.

C'est pourquoi, à ce stade de notre étude, il y a quelques intérêts à faire une différence entre l'œil organe, sa fonction de perception, et à replacer le rapport lisible / visible à l'aune regard. Et c'est en effectuant un détour par la peinture que nous poursuivons notre propos. Cela peut paraître paradoxal tant le tableau, l'art pictural, semblent être à l'envers de l'affiche. D'une part, il est prédiqué par l'acte contemplatif, même si, comme nous allons l'indiquer, le tableau a un effet de capture du regard. D'autre part, le tableau agit sur son spectateur, en renvoyant et réorientant le jeu du lisible et du visible. En effet, lorsque nous regardons un tableau, on observe une organisation du monde. Sur ce point, on peut trouver une similitude entre affiches et tableaux. Il semble en outre important de souligner, que la perspective et ce, dès le $X V^{\text {ème }}$ siècle modifie la perception et représente, pour nous occidentaux, un maître-mot absolu, celui de l'ordre du monde. Il est bien question de visible, mais est-ce tout ?

De fait, nous ne sommes pas dans l'instance de perception médiatisant le sensible et le symbolique, car le corps divisé entre perception, prise en charge symbolique et jouissance, fait écran ; et comme le dit RosePaul Vinceguerra, c'est plutôt un obstacle à la vision. Nous serions selon la psychanalyste frappés ${ }^{25}$ «d'un manque dans l'image spéculaire, impossible à restituer au corps imaginaire (celui que nous imaginons être) car rien ne peut résoudre la scission originelle de l'être humain par son entrée dans le langage». Ainsi, dans une

\footnotetext{
${ }^{24}$ Jacques Lacan, Les 4 concepts fondamentaux de la psychanalyse, Edition du Seuil, 1973 , p.87.

${ }^{25}$ Vinceguerra Rose-Paul, Tu ne me vois pas d'où je te parle, Conférence on line, site www.cause freudienne.org
} 
tentative de combler ce manque, le «chercher à voir toujours plus, et le pousse à voir, en sont deux modes $\gg^{26}$. Le regard, selon Rose-Paul Vinceguerra, est une interception perceptive, «interversion de l'œil et $d u$ spectacle qui fait que dans l'expérience du regard, je me situe moins comme voyant que comme $v u »^{27}$.

C'est pourquoi l'avidité du voir au-delà, de la pulsion du regard, du pousse-à-voir, se perdrait dans la ligne de fuite que représente la ligne d'horizon du tableau. On peut dire que le regard peut se poser dans cet espace voulu par le peintre. Jacques Lacan disait que la fonction de l'art était d'apaiser la pulsion du pousse-à-voir, puisque le peintre permettrait au spectateur de l'œuvre de rencontrer un obstacle. Le peintre proposerait moins une représentation mais plutôt « un quelque chose qu'on ne s'attend pas à voir $\gg^{28}$.

Ces quelques remarques sur la peinture mettent peut-être, en quelque sorte, en lumière, la différence entre tableau et affiche. Nous avons relevé, dans notre analyse l'importance des enjeux de manipulation et de conflit entre les mondes possibles de prédications qu'il met en scène et empêche toute pause du passant. Et si la lecture existe, elle reste par définition fragmentaire. Cela dit, il faut toutefois souligner que l'affiche tout comme le tableau, est une présentation visuelle. On retrouve alors ici aussi, la fonction de visibilité et sa doublure, à savoir un corps mu par la jouissance. Un déplacement du regard, un pousseà-voir, s'opère alors. Notons que contrairement au spectateur d'un tableau, l'activité pulsionnelle du passant n'est guère susceptible d'engendrer l'acte sublimatoire propre au geste pictural d'un peintre. Le peintre peut lui, capturer la pulsion scopique de celui qui observe le tableau. Ce qui est fort différent en ce qui concerne l'affiche.

Dans notre environnement urbain, nous sommes moins absorbés par ce que l'affiche nous donne à lire et à voir, que par l'irruption permanente du pousse-à-voir qu'elle suscite. Ce regard ne peut être apaisé car il n'est que sporadique et répétitif. Nous pourrions même avancer que, sous l'activité perceptive et l'information fragmentée que l'affiche délivre en permanence, il se joue une activé pour rien, sans visée particulière, qui génère une absence de tranquillité sans contours. Ainsi, au terme de l'étude de quelques emplacements d'affichage du passage St Rémi, nous émettons une hypothèse: L'affiche ouvrirait sans cesse une quête d'un encore plus visible dans

\footnotetext{
${ }^{26}$ Ibid.

${ }^{27} \mathrm{Ibid}$

${ }^{28}$ Ibid.
} 
Analyses

le rapport tensif, dans le télescopage voire le conflit entre lisible et visible. On peut également se demander si une des fonctions de l'affichage urbain ne serait pas alors, une activation et une répétition infinie de la pulsion scopique? Dénoué d'un point de butée, par l'affiche, notre regard resterait sans réponse dans l'au-delà du visible, entretenant à vide un regard qui ne voit plus grand-chose, que rien ne peut réellement suspendre.

\section{Bibliographie :}

Fontanille J., (sous la direction de), Les objets aux quotidiens, NAS, édition PULIM, Limoges, 2005.

Lacan J., Les quatre concepts fondamentaux de la psychanalyse, Paris Seuil, 1973.

Mons A., L'affichage et la ville, les métamorphoses entrelacées, in Dans la ville, l'affiche, revue EIDOS, n4, Tours, 1993.

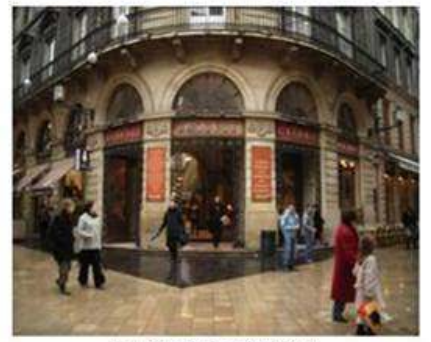

Entrée du Passage St-Rémi

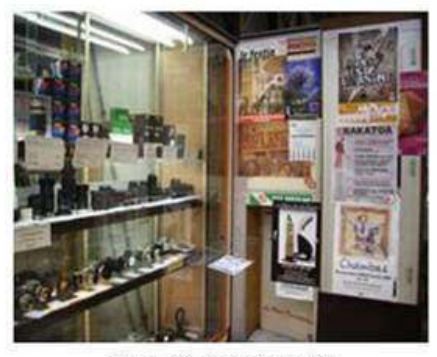

Le magasin de photographes
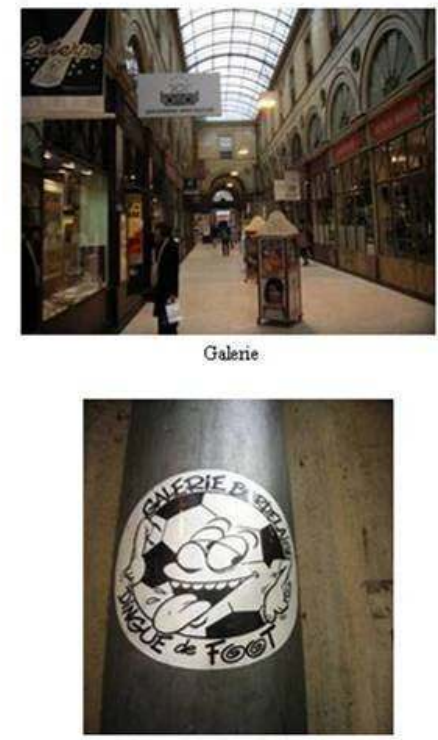

L'autocollant 
